\title{
Author Index to 14th Annual Meeting
}

\author{
Numbers indicate abstract numbers
}

Aarskog, D. 1

Åkerblom, H. K. 2

Allen, T. 36

Andersen, H. 3, 8

Attanasio, A. 4, 43

Audi, L. 5

Aynsley-Green, A. 6

Bachmann, H.-J. 52

Banchini, G. 19

Basmaciogullari, A. 10

Baumann, J. B. 20

Baumann, P. 59

Beckers, C. 58

Beischer, W. 24

Belleville, F. 42

Bernasconi, S. 19

Bertrand, J. 17, 39

Bidlingmaier, F. 7

Bierich, J. R. 29

Binet, E. 9

Binoux, M. 44

Blank, B. 4

Bloom, S. R. 6

Blunck, W. 50, 61

Buchberger, J. 25

Butenandt, 0.7

Cathiard, A. M. 17

Chabanol, F. de 34

Chaussain, J. L. 9

Chiumello, G. 18

Claustrat, B. 13

Clayton, B. 16

Constantinidis, M. 12

Cornette, C. 58

Cser, A. 36

Czernichow, P. 10, 11

Dacou-Voutetakis, C. 12

Dahlen, H. G. 35

Daivd, L. 13, 34

Degenhart, H. J. 30

Dickerman, Z. 14, 65

Dige-Petersen, H. 8

Dillon. M. J. 15

Doron, M. 27

Eeckels, R. 58

Eggermont, E. 58

Eichner, M. 23

Fahlbusch, R. 29

Fairney, A. 16

Fazekas, A. T. A. 26

Fellier, H. 28

Fennell, J. 36

Ferrandez, N. A. 38

Fevang, F. $\phi .1$
Fisher, D. A. 47

Flach, A. 43

Flatau. E. 27

Flynn, D. M. 16

Forest, M. G. 17

Foss, I. 57

Francois, R. 13, 34

Fried, F. 35

Friis-Hansen, B. 3

Friedman, R. II

Gargantini, L. 18

Giovannelli, G. 19

Girard, J. 20, 21

Gleispach, H. 28

Gnehm, H. 22

Golder, W. 49

Grant, D. B. 15

Grignon, G. 42

Grumbach, S. 43

Gupta, D. 4, 23, 29, 43

Hahn, H. 49

Hartemann, P. 42

Heinze, E. 24

Herrmann, H. 26

Holbrook, M. 67

Holder, G. 46

Hollmann, G. 49

Holzer, H. 25

Homoki, J. 26, 35

Hummer, L. 8

Illig, R. 22, 59

Jackson, D. 16

Jacobsen, B. B. 8

Jivani, S. K. M. 46

Jaob, N. 56

Job, J. C. 9

Josefsberg, Z. 27

Käser, H. 25

Kastrup, K. W. 3

Kauli, R. 65

Kaushanski, A. 31

Keller. E. 29

Kellerer, K. 28

Klemm, W. 29

Klфve, H. 1

Knorr, D. 7

Koivukangas, T. 2

Korth-Schütz, S. 66, 68

Kraaipoel, R. J. 30

Kuitunen, P. 40

Landau, P. 67

Laron, Z. 14, 27, 31, 65

Leaf, F. C. 32
Leferink, J. G. 30

Lejeune-Lenain, C. 62

Lehmann, P. 49

Leisti, S. 40

Lenard, G. 53

Lenko, H. L. 33

Levine, L. 68

Levine, L. S. 66, 67

Levinsky, R. J. 15

Locard, E. 34

Malaprade, D. 42

Malvaux, P. 58

Matsaniotis, N. 12

Meder, R. 26, 35

Mildenberger, H. 26

Milner, R. D. G. 36

Morera, A. M. 5

Moschona, M. 12

Nabet, P. 42

New, M. 68

New, M. I. 66

New, N. I. 67

Nilsson, K. O. 37

Nolte, K. 29

Olbing, H. 52

Oliver, C. 11

Ott, F. 59

Pardo, F. J. 38

Parth, K. 64

Paunier, L. 48

Peretti, E. de 39

Perheentupa, J. 33, 40

Petrykowski, W. v. 41

Pfeiffer, E. F. 24

Pham-Huu-Trung, M. T. 44

Pierson, M. 42

Plauchu, H. 13

Prader, A. 22, 59, 63

Prager-Lewin, R. 14, 65

Proeschel, M. F. 44

Putkamer, K. v. 29

Rabinovitch, A. 48

Rager, K. 4, 23, 29, 43

Raux, M. C. 44

Rayner, P. H. W. 46

Roger, M. 45, 56

Rotter, A. 25

Rudd, B. T. 46

Ruitton, A. 34

Ryness, J. M. 15

Sack, -J. 47

Saenger, P. 66, 68 
Saez, J. M. 5

Schlumberger, A. 9

Schneider, P. 48

Schönberg, D. 50

Schwartz, E. 68

Sippell, W. G. 49

Sizonenko, P. C. 48

Soldat, M.-C. 45

Stahnke, N. 50, 61

Steendijk, R. 51

Steins, P. 50

St $\phi \mathrm{a}, \mathrm{K} . \mathrm{F} .1$

Stolecke, H. 52, 63
Stubbe, P. 53

Tea, N. T. 56

Teller, W. M. 24, 26, 35

Thorsen, T. 1

Tietze, H. U. 55

Torresani, T. 22, 59

Toublanc, J.-E. 45, 56

Trygstad, O. 57

Turner, R. C. 6

Vanderschueren-Lodeweyckx, M. 58

Verkasalo, M. 40
Visser, H. K. A. 30

Werder, E. A. 59

Wiebel, J. 60, 61

Wiedemann, E. 68

Williamson, D. H. 6

Willig, R. P. 50, 61

Wisseler, H. M. 61

Wolter, R. 62

Zachmann, M. 21, 22, 59, 63

Zagalak, M. 63

Zimprich, H. 64

Zuppinger, K. 25 